\title{
Téoros
}

Revue de recherche en tourisme

\section{Les parcs thématiques et le tourisme}

\section{Suzanne Chassé}

Volume 12, numéro 3, octobre 1993

Le renouveau des parcs à thèmes

URI : https://id.erudit.org/iderudit/1077928ar

DOI : https://doi.org/10.7202/1077928ar

Aller au sommaire du numéro

Éditeur(s)

Université du Québec à Montréal

ISSN

0712-8657 (imprimé)

1923-2705 (numérique)

Découvrir la revue

Citer ce document

Chassé, S. (1993). Les parcs thématiques et le tourisme. Téoros, 12(3), 2-2.

https://doi.org/10.7202/1077928ar d'utilisation que vous pouvez consulter en ligne.

https://apropos.erudit.org/fr/usagers/politique-dutilisation/ 


\title{
Présentation
}

\section{Les parcs thématiques et le tourisme}

\author{
Suzanne Chassé
}

Le Comité de rédaction de Téoros a cru, a juste titre, qu'il serait intéressant de créer un numéro sur les parcs thematiques. Le theme peut vous sembler bizarre car on est en droit de sinterroger sur l'existence de tels parcs au Québec.

Mais en relevant les elléments sous-jacents au concept du parc thématique, on comprendra mieux son adaptabilité au contexte québécois. Un parc à thèmes est donc:

- un lieu de loisir consacré a la distraction et au jeu (animation):

- un espace clos organisé autour d'un ou de plusieurs themes:

- un site où la vocation commerciale est importante (restauration, boutiques, etc.);

- un lieu concu pour tous les membres d'une famille:

- un aménagement requérant des investissements majeurs.

/l existe donc au Québec. des sites et attraits qui répondent à plusieurs de ces éléments. Les articles présentés dans ce numéro traitent de differents volets de ces parcs thématiques. IIn'y a pas de consensus sur l'approche suivie par les auteurs car chacun y est allé selon ses préoccupations, sa formation ou ses réalisations.

Le premier article, celui de Marie-Janou Lusignan, résume la naissance des parcs; d'un espace naturel que les rois et l'aristocratie européenne redessinent pour leur plaisir, au parc d'attractions et au parc à thèmes crée par Walt Disney en 1955.

Le second article, par Michel Zins, cerne les enjeux stratégiques des parcs en Amérique du Nord et en Europe. Nous apprenons, entre autres, que la course au gigantisme est devenue trop périlleuse et que les parcs de moindre envergure devront, pour survivre, se trouver un positionnement fort, appuyé sur un avantage concurrentiel.

D'ailleurs, l'article de Pierre Rochon et moi-méme définit, de façon plus quantitative, rimportance de certains criteres économiques pour la création de parcs thématiques dans la région de Montréal.

Dans le mème sens, Mario Girard, maire de Granby, souligne d'une facon intéressante les contraintes liées à la création d'un parc thematique. On pourrait tout aussi bien intituler son article, De la volonté de développementau cercle vicieux du financementll!

Les trois articles suivants présentent des approches toutes différentes et traitent le sujet avec un recul interessant.
L'article de Jean-Claude Jay-Rayonet Brigitte Morneaurebatit notre perception, a partir de l'écologie humaine. I/s associent la réussite d'un parc à la profusion et à la concentration de la consommation sur un même site, à la création de thèmescibles concentrés et à la matérialisation du concept de paradis terrestre!

L'approche adoptée par Jean-Paul Thomin tente d'établir un lien entre les institutions muséales et les composantes de base d'un parc thématique à caractère culturel.

Raymond Montpetit, quant a lui, présente des sites qui nous font revivre les événements (living history) plutôt que simplement faire voir et savoir. Ils sont d'ailleurs, en ce sens, plus près des parcs thématiques que des institutions culturelles.

Les derniers articles touchent trois sujets tout a fait différents: celui que jai fait en collaboration avec Promexpo, montre le cheminement d'un concept initial concernant la Cité internationale du Pere Noël jusqu'á celui de la mise en marché des attraits du Québec sous le thème de la NordiCité. L'article de Thierry Coltier est plus près des réalités quotidiennes car, comme gestionnaire de parc, il est préoccupé par la clientele sensible au rapport qualité-prix et cela, dans un contexte concurrentiel.

Enfin, le texte d'Yves Robillard nous amène dans un monde de parcs futuristes. Les notions de fête et de jeu sont associées a des besoins d'expérimenter avec tout san corps plutôt que simplement avec sa tête!

Ce numéro, nous l'espérons, est éclaté: son contenu aborde plusieurs facettes en évitant de se limiter à une approche un peu trop simpliste des parcs thematiques. On perçoit de plus en plus une évolution du concept car les institutions culturelles et muséales, pour accroître leur fréquentation (dans une optique d'autofinancement), doivent se rapprocher des besoins du public (divertissement) tandis que les parcs thematiques doivent se doter d'une plus grande authenticité pour rencontrer les nouveaux besoins de la clientéle (connaissance). Tous deux devront offrir une expérience a la fois culturelle et divertissante et desservir une clientèle plus élargie. 